\title{
Haematuria presenting in outpatients attending a department of genitourinary medicine
}

\author{
K. L. AMARASURIYA \\ From the Department of Genitourinary Medicine, Guy's Hospital, London
}

SUMMARY Of all the patients attending a department of genitourinary medicine during a 10-month period, about $2 \%$ (1 out of 50 ) presented with haematuria, or haematuria was discovered on initial examination. In about $25 \%$ of cases, the haematuria was due to Escherichia coli infection of the lower genitourinary tract. Gonococcal infection was the next commonest cause; one patient with gonorrhoea presented with frank urethral bleeding. In the remaining patients other causes of haematuria, which included renal cyst, carcinoma of the ureter, bilharziasis, and IgA disease, required more extensive investigations and follow up.

\section{Introduction}

The spectrum of disease in a department of genitourinary medicine covers infective and noninfective disorders. Since these departments were for the most part established for the clinical investigation of infectious disease, it is of interest to report the incidence of haematuria which occurred in patients attending over a 10-month period. None of these patients had been investigated elsewhere. This paper describes the type of presentation and the investigations undertaken in 12 such cases.

\section{Methods}

The basic investigations which were carried out in each case were: urethral smear (for Gram staining and microscopy); urethral culture (for detection of gonococci and anaerobic bacteria); mid-stream urine analysis (for detection of casts, leucocytes, red blood cells, protein, and glucose) and culture for microorganisms; centrifuged deposit of urine (for Gram staining and culture). The following investigations were done in some cases, if indicated: intravenous urography (IVU), urethroscopy, serum biochemical analysis, and ASO titre. In special cases, urine was examined for tubercle bacilli or Bilharzia.

\section{Results}

The causes of haematuria in the 53 patients investigated are given in the table.

Address for reprints: K. L. Amarasuriya, Department of
Genitourinary Medicine, Guy's Hospital, London SE1 Received for publication 21 August 1978
Table Causes of haematuria in 53 patients investigated

\begin{tabular}{ll}
\hline Cause & No. of cases \\
\hline Escherichia coli urinary infection & 25 \\
Gonorrhoea & 7 \\
Abacterial urethritis & 6 \\
Renal calculi & 4 \\
Mycoplasmal urethritis & 3 \\
Tumour & 2 \\
Cryptogenic & 2 \\
Renal cyst & 1 \\
Ureterocoele & 1 \\
Tuberculosis & 1 \\
Bilharziasis & 1 \\
\hline
\end{tabular}

\section{CASE HISTORIES}

Case 1

A married Englishman, aged 23, presented with a history of haematuria and urethral discharge for one day. He cohabited with his wife. Clinical findings were: purulent and blood-stained urethral discharge; Neisseria gonorrhoeae detected in urethral smear and culture; urine analysis showed first and second glass haze and $\mathrm{RBC}+$; and mid-stream urine showed $\mathrm{WBC}+, \mathrm{RBC}+$, and no growth on culture. The patient was treated with $2 \cdot 4$ megaunits procaine penicillin intramuscularly plus $2 \mathrm{~g}$ oral probenecid and made an uneventful recovery. Urethral gonorrhoea was diagnosed.

Case 2

A married Englishman, aged 46, presented with a history of haematuria and urethral discharge for three days. He had had marital intercourse five days previously and intercourse with an unknown girl three weeks previously. Clinical findings were: purulent urethral discharge and pus cells $(++)$ in urethral smear; blood-stained urine showing first and second glass haze with $\operatorname{RBC}(+)$ and protein $(+)$; and 
centrifuged urine deposit containing pus cells $(+)$ and coliform organisms on Gram stain; mid-stream urine showed

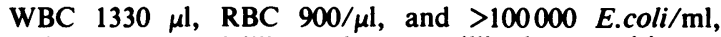
resistant to ampicillin and amoxycillin but sensitive to doxycycline, gentamicin, and trimethroprim. The patient responded satisfactorily to treatment with triple tetracycline. E.coli infection of the lower genitourinary tract was diagnosed.

\section{Case 3}

A married Bangladeshi man, aged 55, presented with a history of haematuria and urethral discharge since the previous night, together with fever and rigors. He had had no sexual intercourse for five years. Coincidental findings were: temperature $38.5^{\circ} \mathrm{C}$; purulent and bloody urethral discharge with coliform organisms on Gram stain; urine showed second glass haze and a positive result for blood on routine analysis; full blood count showed increased WBC with neutrophilia, ESR $19 \mathrm{~mm}$ in first hour; and midstream urine specimen contained WBC $460 / \mu 1$, RBC $400 / \mu \mathrm{l}$, and $100000 \mathrm{E} . \mathrm{coli} / \mathrm{ml}$, sensitive to amoxycillin, ampicillin, gentamicin, trimethoprim, and doxycycline. Intravenous urography was normal. The patient was treated with amoxycillin, $500 \mathrm{mg}$ four times daily for one week, and became asymptomatic. E.coli infection of the lower genitourinary tract, with constitutional symptoms, was diagnosed.

\section{Case 4}

A single Englishman, aged 17, presented with a history of haematuria and of urethral discharge for two weeks, for which he had been given Septrin by his own doctor. He had had sexual intercourse with an unknown girl four weeks previously. Clinical findings were: purulent urethral discharge; urine showed second glass haze with blood $(\mathrm{RBC}+)$ and protein (a trace); and mid-stream urine specimen contained WBC $3690 / \mu \mathrm{l}$, RBC $480 / \mu$ l but no growth on culture. Large-colony mycoplasmas were found on culture from urethral discharge. No abnormality was found in full blood count, ESR, ASO titre, or throat swab. Intravenous urography suggested a small cyst in the left kidney but was otherwise normal. Ultrasonic scan suggested a small cyst in the upper pole of the left kidney. The patient recovered after treatment with triple tetracycline and was referred to the genitourinary surgeon. Renal cyst and mycoplasma-positive urethral discharge was diagnosed.

\section{Case 5}

A single Rhodesian man, aged 20, presented with haematuria and urethral discharge. He gave a history of bilharziasis (vesical) treated three years earlier in Rhodesia, where he had lived until three months before presenting here. Clinical findings were: purulent urethral discharge containing coliform organisms on Gram stain; bloodstained urine showed first glass haze and second glass haze; centrifuged urine deposit contained WBC, RBC, and coliforms; and mid-stream urine showed WBC 14/ $\mu$, RBC $200 / \mu$ l, but no growth of micro-organisms. Microscopical examination of terminal strained urine gave negative results for Bilharzia, ova, and cysts. The following investigations all gave negative results or were within normal limits: full blood count and ESR, blood urea and serum electrolytes, ASO titre, throat swab, Mantoux $1 / 10000, x$-ray examination of chest, and intravenous urography. The patient was treated with triple tetracycline, and a week later the discharge had cleared and prostatic examination was normal; the haematuria (on microscopical examination), however, persisted. Further laboratory examination of strained terminal urine demonstrated the presence of Schistosoma ova. Cystoscopy was advised, but the patient left the country without keeping the appointment and did not return. Bilharziasis with haematuria was diagnosed.

\section{Case 6}

A single Englishman, aged 29, presented with a history of urethral discharge and haematuria for three days, and passing renal stones intermittently for the past three years. He had sexual contact only with his wife. There was a previous history of coeliac disease in childhood, treated with an appropriate diet, and of pulmonary tuberculosis in 1958, treated in Ireland. His mother had had tuberculosis, his father diabetes and renal stones, and his sister renal stones. Urine examination showed haematuria on microscopical examination, no acid-fast bacilli on Ziehl Neelson stain, and no bacteria on culture after eight weeks incubation on several occasions. Mid-stream urine specimens contained no WBC; RBC was $200 / \mu$; no growth of organisms occurred on culture. Blood urea and serum electrolytes, urine oxalate, and blood glucose were all within normal limits. Intravenous urography showed renal calculi in the left kidney, and the patient was referred to a surgeon. There was no sign of tuberculosis. Renal calculi were diagnosed.

\section{Case 7}

A single West Indian man, aged 21, presented with a history of haematuria and urethral discharge for five days. Clinical findings were: purulent urethral discharge; blood-stained urine showing first glass haze with shreds and second glass haze with RBC; and the centrifuged deposit contained WBC. Ureaplasma urealyticum and Mycoplasma hominis but no chlamydiae were isolated from the urethral discharge. Mid-stream urine showed no growth of organisms. This patient's condition cleared normally on treatment with triple tetracycline. Endoscopy was reported as normal. Mycoplasma-positive urethritis was diagnosed.

\section{Case 8}

A married Englishman, aged 26, presented with a history of lower backache for two months and dark urine for four days. He cohabited with his wife. On examination, there was no urethral discharge. Urine showed first glass haze and second glass haze with RBC; centrifuged deposit contained no WBC and no organisms. The following investigations were all normal: mid-stream urine, full blood count and ESR, ASO titre, and throat swab. The IVU showed a large 'spring onion' defect in the bladder on the right side, indicating a large ureterocoele which was causing obstrucion to the entire collecting system on that side and resulting in haematuria. The left kidney was normal, but there was some loss of cortex on the right side. The patient was referred to a genitourinary surgeon. A ureterocoele was diagnosed. 


\section{Case 9}

A married Englishman, aged 66, presented with a history of periodic epigastric pain and a dull, almost continuous, mainly right-sided abdominal pain radiating to the back for the past two years. On examination, there was no urethral discharge. Blood pressure reading was $140 / 80 \mathrm{mmHg}$. Urine showed first glass clear and second glass clear with microscopic haematuria. MSU contained no WBC, RBC $130 / \mu \mathrm{l}$, and no growth of organisms. Full blood count and ESR, blood urea, serum electrolytes, serum amylase, ASO titre, and throat swab were all normal. Barium meal examination showed a healed duodenal ulcer. The IVU showed a lesion of the right ureter; this proved to be a carcinoma which was removed surgically and the ureter transplanted into the bladder. The haematuria cleared and the patient made an uneventful recovery. Carcinoma of the ureter was diagnosed.

\section{Case 10}

A single English boy, aged 15, was referred from the urology department with a history of persistent haematuria over the past three years and occasional frank bleeding. There had been intermittent dysuria for three months. There was no history of sexual intercourse and no history of masturbation. On examination, there was a clear urethral discharge and a small abrasion on the inner, left aspect of the meatus. The urethral smear contained pus cells. No mycoplasmas or chlamydiae were isolated. Darkground microscopy showed negative results for treponemes and Trichomonas vaginalis. Herpes virus was cultured from the meatal lesion. The first glass urine was clear with shreds and the second glass was clear with RBC. Mid-stream urine contained WBC $14 / \mu \mathrm{l}$, RBC $20 / \mu \mathrm{l}$, but no growth on culture. No abnormality was found on IVU, micturating cystogram, urethroscopy, cystoscopy, or prostatic examination. Full blood count and ESR, blood urea and serum electrolytes, ASO titre, and throat swab were all within normal limits. The non-specific urethritis responded to treatment with triple tetracycline, but the microscopic haematuria persisted over the following two months and was considered to be due to capillary bleeding in the posterior or membranous urethra. Non-specific urethritis (not sexually mediated) and cryptogenic haematuria were diagnosed.

\section{Case 11}

A single Englishman, aged 20, presented with a history of haematuria for three days together with bilateral aches in the loins. On examination there were no lower genitourinary tract signs apart from macroscopic and microscopic haematuria. His blood pressure was normal, and there was no history of previous streptococcal infection or of present or previous joint involvement. He had been seen in this clinic six weeks previously with non-specific urethritis. He also gave a history of haematuria nine months previously, which was investigated by his general practitioner and confirmed by mid-stream urine. An IVU performed at that time was normal. Investigations on this occasion showed: IVU normal; mid-stream urine specimen containing RBC but no growth of organisms. The following tests all gave negative results or were within normal limits: ASO titre, throat swab, autoantibodies, urine cultures for tubercle bacilli successively, blood urea and electrolytes, prothrombin and clotting time, full blood count and ESR, Paul Bunnell, $x$-ray examination of chest, and ECG. Renal biopsy showed a mild mesangial proliferative glomerulonephritis with IgA deposits. It is of interest that a similar patient, in whom IgA disease was also diagnosed, presented during the previous year in this department. IgA disease was diagnosed.

\section{Case 12}

A single African man, aged 24, presented with a urethral discharge and dysuria. He had had sexual intercourse only with a regular consort. Clinical findings were: purulent urethral smear; first and second glass urine contained haze, shreds, and RBC; and centrifuged deposit contained pus cells. Mid-stream urine analysis showed no growth of organisms. No bacterial pathogens, no chlamydiae, and no mycoplasmas were isolated on urethral culture. The condition cleared after a five-day course of triple tetracycline. Abacterial haematuria was diagnosed.

\section{Discussion}

The manifold causes of haematuria are well known (Hart, 1973; Lytton, 1977; Higgins, 1978). Lee and Davis (1953) found that most of the 1000 cases of haematuria investigated by them could be classified under six common causes (in descending order of frequency): cystitis, tumour of the bladder, benign hypertrophy of the prostate, chronic prostatitis, essential (of doubtful aetiology) haematuria, and calculus of the ureter. According to Boyd (1977) three common causes-namely, renal and urothelial malignancy, infection, and calculus disease-together accounted for most diagnoses while most of the remaining cases of haematuria were due to glomerulonephritis, renal cystic disease, trauma, infarction, and embolism. Lytton (1977) states that in his experience 'the commonest cause of haematuria is acute cystitis'.

It is of interest that out of the many female patients attending this department with E.coli infection of the lower genitourinary tract during the period of the present study none presented with haematuria whereas over three-quarters of the male patients with E.coli infection did have haematuria. Where blood was found in the urine of female patients, in almost every case it was due to contamination (for example, menstruation). In one female patient, however, who presented with vaginal discharge and haematuria the latter was due to urethral gonorrhoea.

The case of bilharziasis reported above is a reminder that, especially in these days of increased travel, such conditions need to be considered as possible causes of haematuria. 


\section{References}

Boyd, P. J. R. (1977). Haematuria. British Medical Journal, 2, 445446.

Hart, F. D. (editor) (1973). French's Index of Differential Diagnosis, tenth edition, pp 306-307. Wright: Bristol.

Higgins, P. M. (1978). Haematuria. British Journal of Hospital Medicine, 19, 325-334.
Lee, L. W. and Davis, E. (1953). Gross urinary hemorrhage: symptom not disease. Journal of the American Medical Association, 153, 782-784.

Lytton, B. (1977). Bleeding from the urinary tract. Medical Times, 195, 27-35. 